\title{
Sphingolipid Metabolism is Strikingly Different Between Pollen and Leaf in Arabidopsis as Revealed by Compositional and Gene Expression Profiling
}

\author{
Kyle D. Luttgeharm ${ }^{1 \ddagger}$, Athen N. Kimberlin ${ }^{1 \ddagger}$, Rebecca E. Cahoon ${ }^{1}$, Ronald L. Cerny ${ }^{2}$, \\ Johnathan A. Napier ${ }^{3}$, Jonathan E. Markham ${ }^{1}$, and Edgar B. Cahoon ${ }^{1 *}$
}

${ }^{1}$ Center for Plant Science Innovation and Department of Biochemistry, E318 Beadle Center, 1901 Vine Street, University of Nebraska-Lincoln, Lincoln, NE 68588, USA

${ }^{2}$ Department of Chemistry, 710 Hamilton Hall, University of Nebraska-Lincoln, Lincoln, NE 68588, USA

${ }^{3}$ Department of Biological Chemistry and Crop Protection, Rothamsted Research, Harpenden, AL5 2JQ, United Kingdom

fThese authors contributed equally to this work.

*Corresponding Author:

Edgar B. Cahoon

E318 Beadle Center

1901 Vine Street

University of Nebraska-Lincoln

Lincoln, NE 68506 USA

Phone:_+1 4024725611

Email: ecahoon2@unl.edu

Author emails: Kyle Luttgharm (kyle.luttgeharm@huskers.unl.edu), Athen Kimberlin (akimberlin1@ huskers.unl.edu), Rebecca Cahoon (rcahoon2@unl.edu), Ronald Cerny (rcerny1@unl.edu), Johnathan Napier (johnathan.napier@ rothamsted.ac.uk), Jonathan Markham (jemarkham@unl.edu), Edgar Cahoon (ecahoon2@unl.edu) 


\begin{abstract}
Although sphingolipids are essential for male gametophytic development in Arabidopsis thaliana, sphingolipid composition and biosynthetic gene expression have not been previously examined in pollen. In this report, electrospray ionization (ESI)-MS/MS was applied to characterization of sphingolipid compositional profiles in pollen isolated from wild type Arabidopsis Col-0 and a long-chain base (LCB) $\Delta 4$ desaturase mutant. Pollen fractions were highly enriched in glucosylceramides (GlcCer) relative to levels previously reported in leaves. Accompanying the loss of the $\Delta 4$ unsaturated LCB sphingadiene (d18:2) in the $\Delta 4$ desaturase mutant was a 50\% reduction in GlcCer concentrations. In addition, pollen glycosylinositolphosphoceramides (GIPCs) were found to have a complex array of N-acetylglycosylated GIPCs, including species with up to three pentose units that were absent from leaf GIPCs. Underlying the distinct sphingolipid composition of pollen, genes for key biosynthetic enzymes for GlcCer and d18:2 synthesis and metabolism were more highly expressed in pollen than in leaves or seedlings, including genes for GlcCer synthase $(G C S)$, sphingoid base $\mathrm{C}-4$ hydroxylase 2 (SBH2), LCB $\triangle 8$ desaturases (SLD1 and SLD2), and LOH2 ceramide synthase ( $\mathrm{LOH} 2)$. Overall, these findings indicate strikingly divergent sphingolipid metabolism between pollen and leaves in Arabidopsis, the significance of which remains to be determined.
\end{abstract}

Keywords: Arabidopsis thaliana; Brassicaceae; pollen, sphingolipid, lipidomics, glucosylceramide, glycosylinositolphosphoceramide 


\section{Introduction}

Sphingolipids are major structural components of the plasma membrane, tonoplast, and endomembranes and are enriched in detergent-resistant plasma membrane microdomains or lipid rafts in plant cells (Borner et al., 2005; Mongrand et al., 2004; Sperling et al., 2005).

Desaturation of sphingolipid long-chain bases (LCBs) as well as total sphingolipid levels have been demonstrated to contribute to cold-tolerance (Chen et al., 2008; Guillas et al., 2012; Nagano et al., 2014). Sphingolipid metabolites also function in non-structural roles in plants. The accumulation of ceramides and free long-chain bases (LCBs), for example, has been shown to trigger programmed cell death (PCD) through a MAP kinase 6 transduction pathway, which is important for hypersensitive response resistance to pathogens (Brodersen et al., 2002; SaucedoGarcía et al., 2011). Phosphorylated long-chain bases have also been shown to participate in ABA signaling for guard cell closure (Coursol et al., 2003; Coursol et al., 2005; Ng et al., 2001) and cold-responsive nitric oxide production has been linked to reductions in phosphorylated ceramides and LCBs levels (Cantrel et al., 2011).

Sphingolipids are defined by the presence of long-chain bases (LCBs), which are linked through an amide bond to fatty acids to form ceramides, the backbones of complex sphingolipids. Ceramides can be modified by addition of polar head groups consisting of phosphates, carbohydrates, or combinations of the two (Chen et al., 2010; Lynch and Dunn, 2004; Markham and Jaworski, 2007). Additionally LCBs can be modified by hydroxylation at their C-4 positions to yield trihydroxy LCBs and/or desaturation at the C-4 $(\Delta 4)$ and C-8 $(\Delta 8)$ positions (Lynch and Dunn, 2004). Further structural diversity in sphingolipids is generated by hydroxylation of the $\mathrm{C}-2$ ( or $\alpha$ ) position of the constituent fatty acids, which typically range in chain-lengths from 16 to 26 carbon atoms (Chen et al., 2010; Markham et al., 2011). Moreover, fatty acids in sphingolipids of Arabidopsis, other Brassicaceae and many Poaceae can contain $\omega$ 9 unsaturation (Imai et al., 2000).

Contributing to the large structural complexity of plant sphingolipids is an array of possible polar head groups linked to the $\mathrm{C}-1$ hydroxyl group of ceramides. In Arabidopsis, two major classes of complex sphingolipids occur: glucosylceramides (GlcCer) and glycosylinositolphosphoceramides (GIPCs). In contrast to the simple glucose head group of GlcCer, GIPCs can contain an array of sugar residues linked to an inositol phosphate residue than is present in all GIPCs. Recently Bure et al. (2011) developed a provisional form of GIPC 
nomenclature based on the numbers and composition of the head group sugars: Hex-HexA-IPC (Series A), Hex-Hex-HexA-IPC (Series B), Pent-Hex-Hex-HexA-IPC (Series C), (Pent) ${ }_{2}$-HexHex-HexA-IPC (Series D), (Pent) ${ }_{3}$-Hex-Hex-HexA-IPC (Series E), (Pent) ${ }_{4}$-Hex-Hex-HexA-IPC (Series F). In this nomenclature scheme, Hex corresponds to a hexose sugar, Pent corresponds to a pentose sugar, HexA corresponds to hexuronic acid, and IPC corresponds to inositolphosphoceramide. The primary GIPC head group identified to date in Arabidopsis leaves contains a single hexose (Hex) with hydroxylation $(\mathrm{OH})$ bound to a hexuronic acid (HexA) linked to IPC and corresponds to that of Series A (Buré et al., 2011; Bure et al., 2014; Cacas et al., 2013; Markham and Jaworski, 2007). Other plants, such as tobacco and tomato, contain large amounts of Hex with N-acetylation (NAc)-HexA-IPCs with up to seven sugar residues bound to IPC (Buré et al., 2011; Bure et al., 2014; Hsieh et al., 1981; Kaul and Lester, 1978; Markham et al., 2006; Tellier et al., 2014). For example, GIPCs in tobacco BY2 cells contain up to four Pent and three Hex residues (including glucuronic acid, HexA) linked to IPC (Buré et al., 2011). Although $\operatorname{Hex}(\mathrm{OH})-\mathrm{HexA}$ (Series A) is the primary glycosylation of GIPCs in Arabidopsis leaves, additional GIPC structures including $\operatorname{Hex}-\mathrm{Hex}(\mathrm{OH})-\mathrm{HexA}-\mathrm{IPC}$ (Series B), (Pent) $)_{2}$-Hex-Hex(OH)-HexA-IPC (Series D), (Pent) ${ }_{3}$-Hex-Hex(OH)-HexA-IPC (Series E), and

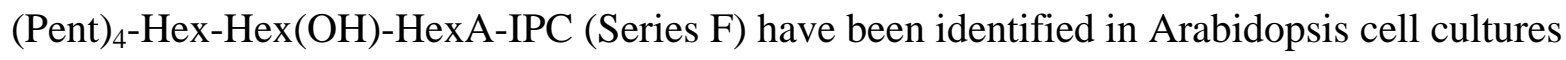
(Buré et al., 2011; Mortimer et al., 2013). A recent study also found Hex(NAc)-HexA-IPCs (Series A) in Arabidopsis seeds and seedlings (Tellier et al., 2014), not previously found in Arabidopsis cell cultures (Buré et al., 2011). The GIPCs of Arabidopsis seeds and seedlings, however, lacked the complex sugar head groups previously found in Arabidopsis cell culture (Buré et al., 2011; Tellier et al., 2014). The functional significance of the different GIPC sugar structures is currently unknown as is the reason for their occurrence in only certain cell types.

Sphingolipids are essential for pollen development in Arabidopsis. In this regard, null mutants for the LCB2 subunit of serine palmitoyltranferase, which catalyzes the first step in sphingolipid LCB synthesis, have non-viable pollen (Dietrich et al., 2008; Teng et al., 2008). In addition, double mutants of the redundant $L C B 2 a$ and $L C B 2 b$ genes in Arabidopsis were unable to transmit the mutant loci through pollen, and pollen lacking LCB biosynthetic ability displayed aberrant endomembranes and lacked the Golgi-derived intine layer (Dietrich et al., 2008). In addition, a T-DNA insertion mutant of ssSPTa encoding the major stimulatory small subunit of SPT results in defective pollen development (Kimberlin et al., 2013). 
Arabidopsis differs from most plant species in that expression of the gene for LCB $\Delta 4$ desaturase (At4g04930) is limited almost exclusively to pollen (Islam et al., 2012; Michaelson et al., 2009). As a result, LCBs with $\Delta 4$ unsaturation are enriched in pollen and flowers, but are nearly absent in leaves of Arabidopsis (Michaelson et al., 2009). Mutants defective in LCB $\Delta 4$ desaturation, however, lack detectable defects in pollen development (Michaelson et al., 2009). LCB $\Delta 4$ unsaturation is found exclusively in the trans configuration, and typically in combination with either cis or trans $\Delta 8$ unsaturation in the C18 dihydroxy LCB sphingadiene (d18:2) (Sperling et al., 1998). In addition, $\Delta 4$ unsaturated LCBs are found in GlcCers, but largely absent from GIPCs (Michaelson et al., 2009; Sperling et al., 2005).

Despite the fact that sphingolipids are essential for pollen and that the occurrence of the LCB d18:2 is limited primarily to pollen in Arabidopsis, a comprehensive profiling of pollen sphingolipids has not been previously described. This report provides a comprehensive description of the sphingolipid composition of Arabidopsis pollen and compares it to that of Arabidopsis leaves from numerous prior reports (Chen et al., 2012; Chen et al., 2008; Kimberlin et al., 2013; Markham and Jaworski, 2007; Markham et al., 2011). In addition to characterization of pollen from wild-type Col-0, pollen from a T-DNA mutant of the single LCB $\Delta 4$ desaturase gene (At4g04930) was also examined (Michaelson et al., 2009) to gain further insights into the importance of $\Delta 4$ unsaturated LCBs in pollen sphingolipid metabolism. In addition, RNA-Seq and microarray data for expression of key sphingolipid biosynthetic genes in Arabidopsis pollen and leaves was compiled, highlighting the differences in sphingolipid metabolism between the two tissue types. Collectively, these data show large differences in sphingolipid composition and biosynthetic gene expression between pollen and leaves, including the identification of an array of abundant novel GIPC structures in pollen indicating that sphingolipids may play a unique role in pollen.

\section{Results}

\subsection{Pollen isolation}

Two methods for pollen isolation were compared: a vacuum-based method and a buffered mannitol-based method (Honys and Twell, 2003; Johnson-Brousseau and McCormick, 2004). The latter method resulted in higher yields and more rapid recovery of pollen. Given the need for significant amounts of pollen with minimal lipolytic degradation for sphingolipid profiling, 
the buffered-mannitol method was chosen for use in these studies. Using this method, highly enriched, intact pollen was isolated that contained only small amounts of lysed pollen (as determined by viability staining) and floral tissue (Figure 1A, B). RT-PCR of the enriched pollen also revealed expression of the LCB $\triangle 4$ desaturase $(\triangle 4 D E S)$ gene, a pollen-specific gene in Arabidopsis (Figure 1C, D). Lyophilized pollen isolated from the Arabidopsis Col-0 and a mutant of the LCB $\triangle 4$ DES mutant plants were subsequently used for ESI-MS/MS profiling of sphingolipid content and composition.

\subsection{Arabidopsis Col-0 pollen sphingolipidome}

As has been previously reported (Markham and Jaworski, 2007) and confirmed in other studies (Chen et al., 2012; Chen et al., 2008; Markham et al., 2011), the sphingolipidome of Arabidopsis Col-0 leaves consists primarily of provisionally identified $\mathrm{Hex}(\mathrm{OH})-\mathrm{HexA}-\mathrm{IPC}$ (Series A) and glucosylceramide, which occur at a molar ratio of approximately $2: 1$. In addition, ceramides, hydroxyceramides (i.e., ceramides containing 2-OH fatty acids), free LCB, and LCB-phosphates account for $\leq 10 \%$ of the sphingolipids of Arabidopsis leaves (Markham and Jaworski, 2007). In addition, the LCBs in Arabidopsis leaves consist almost entirely of t18:1, t18:0, d18:1, and d18:0 (Chen et al., 2006; Markham et al., 2006). The sphingolipid profile of Arabidopsis Col-0 pollen determined in this study was strikingly different than that of Arabidopsis leaves (Figure 2). Among these differences, the glucosylceramide content was nearly 8-fold higher than that reported in Arabidopsis leaves ( 1,377 nmol/g in pollen vs. $160 \mathrm{nmol} / \mathrm{g}$ in leaves) (Markham and Jaworski, 2007). Consistent with this, nearly 50\% of the LCBs in pollen GlcCer were d18:2, which was not detectable in Arabidopsis Col-0 leaf as reported previously (Markham and Jaworski, 2007). In addition, free ceramides, hydroxyceramides, and free LCBs were found to be more abundant in pollen on a per gram dry weight basis than in leaf (Markham and Jaworski, 2007).

The only sphingolipid found to be less abundant in pollen relative to leaf was GIPC, specifically the provisionally identified $\operatorname{Hex}(\mathrm{OH})$-HexA-IPC GIPC (Series A) found in leaf. All previous studies of Arabidopsis leaf sphingolipids have found GIPCs as the most abundant sphingolipid class (Chen et al., 2012; Chen et al., 2008; Markham and Jaworski, 2007; Markham et al., 2011) so it was surprising to find that $\operatorname{Hex}(\mathrm{OH})$-HexA-IPC GIPC (Series A) was five-fold lower in pollen. One hypothesis to explain this apparent reduction in GIPC levels is that pollen 
synthesizes other GIPC types with alternative glycosylation patterns. In order to test this hypothesis, a precursor scan using product ion $662.60 \mathrm{~m} / \mathrm{z}$ (corresponding to the t18:1_h24:1 ceramide fragment) was performed. This identified ions indicative of Hex(NAc)-HexA-IPCs (Series A) with the addition of multiple sugar residues (Figure 3), as well as the provisionally identified leaf-type $\mathrm{Hex}(\mathrm{OH})$-HexA- IPC (Series A), predicted and observed mass for detected species can be found in Supplemental Table 1. To further confirm the identities of these ions, MRMs were developed for complex GIPCs not previously reported in Arabidopsis. These analyses confirmed the presence of provisionally identified (Pent) ${ }_{3}$-Hex-Hex(NAc)-HexA(Series E), (Pent) $)_{2}-H e x-H e x(N A c)-H e x A-(S e r i e s ~ D)$, and Hex-Hex(OH)-HexA-IPCs (Series B) (Figure 4A-D) in sphingolipid extracts from pollen. Only the Hex-Hex $(\mathrm{OH})$-HexA-IPC form was found in both leaf and pollen. Notably, the Hex(NAc) GIPCs were found in pollen but were absent from leaves. By using relative quantitation of $\mathrm{Hex}-\mathrm{Hex}(\mathrm{OH})-\mathrm{HexA}-\mathrm{IPC}$, it was found that this species is $\sim$ six-fold more abundant in leaf than in pollen. The lack of standards precluded absolute quantification and full identification of the novel GIPC forms, however, it is possible that their inclusion would result in total GIPC abundance in pollen equivalent to that of leaf.

\subsection{Sphingolipidome of pollen from a LCB $\Delta 4$ desaturase mutant}

While T-DNA disruption of the LCB $\triangle 4 D E S$ gene does not result in observable phenotypic alterations in plant growth or pollen viability (Michaelson et al., 2009), significant changes in the sphingolipidome of pollen from this mutant were found. The most striking change was an approximately 50\% decrease in GlcCer levels relative to pollen from Col-0. However, aside from a lack of the $\Delta 4$ unsaturated LCB d18:2, GlcCer molecular species in pollen of the $\Delta 4$ DES mutant were similar to those in pollen from Col-0 plants (Table 1). Although the detectable differences in GlcCer compositions were small, there were significant increases in the amounts of d18:1_h16:0 (p=0.0092), d18:1_24:1 (p=0.00060) t18:1_h16 (p=0.021), and t18:1_26:0 $(\mathrm{p}=0.013)$. The other major difference between pollen from the $\triangle 4$ DES mutant and Col-0 was an increased amount of the $\operatorname{Hex}(\mathrm{OH})$-HexA-IPC (Series A), which was detected in pollen from $\Delta 4$ DES mutant ( $\mathrm{p}=0.039$ ), resulting from an increased amount of ceramides containing the $\mathrm{d} 18: 0$, t18:0, and t18:1 LCBs (Figure 5B). In addition, free LCB levels were significantly decreased in pollen from the $\triangle 4 D E S$ mutant ( $<0.001$ ), derived, not only from a lack of d18:2 species, but 
also a significant $(\mathrm{p}=0.011)$ decrease in $\mathrm{d} 18: 1$. Overall amounts of sphingolipids were not significantly different in pollen from Col-0 and $\triangle 4$ DES mutant plants (Figure 5C).

\subsection{Gene expression data mining of sphingolipid biosynthetic genes in pollen relative to seedling and leaf}

Previously published RNA-Seq data (Loraine et al., 2013) and the microarray-based Arabidopsis EFP Browser (Winter et al., 2007) were mined for sphingolipid biosynthesis-related genes. Expression levels were obtained for genes encoding the SPT subunits LCB1 (At4g36480), LCB2a (At5g23670), LCB2b (At3g48780), the two 3-ketosphinganine reductases TSC10A (At5g19200) and TSC10B (At3g06060), the two sphingoid base C-4 hydroxylases SBH1 (At1g69640) and SBH2 (At1g14290), the ceramide synthases LOH1 (At3g25540), LOH2 (At3g19260), and LOH3 (At1g13580), LCB $\Delta 4$ desaturase (At4g04930), the two LCB $\Delta 8$ desaturases SLD1 (At3g61580) and SLD2 (At2g46210), glucsoylceramide synthase (GCS; At2g19880), the three IPC synthases (IPS) IPS1 (At3g54020), IPS2 (At2g37940), and IPS3 (At2g29525). RNA-Seq data were also compiled for the recently identified small subunits of SPT (ssSPT) ssSPTa_(At1g06515) and ssSPTb (At2g30942) as well as the recently identified UDP-glucose IPC transferase (IPUT1; At5g18480) (Rennie et al., 2014), which were not present in microarray data in the EFP Browser. The RNA-Seq study contained data for Arabidopsis pollen versus seedling (Loraine et al., 2013), while data for pollen versus leaf was mined from the EFP Browser. Data from RNA-Seq (Figure 6) and microarray data (Supplemental Figure 1) indicated expression of selected genes at higher levels in pollen compared to seedling or leaf. These included $L C B 2 a L C B 2 b, S L D 1, S L D 2$, and $S B H 2$ as well as genes associated with d18:2 and GlcCer synthesis $\triangle 4 D E S$ and GCS. Microarray data also indicated higher pollen expression of the gene for the $\mathrm{LOH} 2$ ceramide synthase that generates ceramides with $\mathrm{C} 16$ fatty acids found primarily in GlcCer. Quantitative PCR (qPCR) conducted to confirm this, revealed 14-fold higher expression of $\mathrm{LOH} 2$ in pollen relative to leaf of Col-0 plants (Supplemental Figure 2). Overall, these data are consistent with an increased GlcCer biosynthetic capacity in pollen, as indicated by sphingolipid profiling.

\section{Discussion}


Sphingolipids are essential for Arabidopsis pollen development, based on studies of serine palmitoyltransferase that catalyzes the first step in LCB synthesis (Dietrich et al., 2008; Kimberlin et al., 2013). Despite this, sphingolipid composition of Arabidopsis pollen has not been previously been examined, nor have expression profiles of sphingolipid biosynthetic genes in Arabidopsis pollen been cataloged. As part of an effort to understand the function of sphingolipids in Arabidopsis pollen, ESI-MS/MS methodology (Markham and Jaworski, 2007) was applied to characterize sphingolipids in Arabidopsis pollen. In addition, extracted data from publicly available RNA-Seq and microarray studies on expression levels of genes for key sphingolipid biosynthetic and LCB modification enzymes were also examined.

One of the most striking findings was the high content of GlcCer in Arabidopsis Col-0 pollen. GlcCer content was approximately 8 -fold higher in pollen than previously reported in leaves of Arabidopsis rosettes, and $\triangle 4$-unsaturated d18:2 isomers composed $>50 \%$ of the GlcCer LCB. These LCB were not detectable in GlcCer from pollen of the LCB $\triangle 4 D E S$ mutant, and GlcCer concentrations were two-fold lower than those in wild-type pollen. This is consistent with the $25 \%$ lower GlcCer levels reported in flowers of the LCB $\triangle 4$ DES mutant relative to flowers of wild-type Col-0 (Michaelson et al., 2009). Given that pollen from the $\triangle 4 D E S$ mutant were shown to be unaffected in germination and morphology (Michaelson et al., 2009), the functional significance of the relative enrichment of GlcCer in Arabidopsis pollen is not clear. It is possible that any selective advantage of GlcCer enrichment in pollen may not be apparent under optimized growth conditions but is instead important for pollen performance, for example, under environmental extremes or for extended viability.

Another distinctive feature of Arabidopsis pollen sphingolipid composition was the unexpected complexity of GIPCs. Initially, complex but incompletely identified GIPCs containing up to six sugar residues were identified by LC-MS precursor scans monitoring for all GIPCs with the t18:1_h24:1 ceramide backbone. This method is limited in its ability to accurately determine the GIPC profile, since in source fragmentation during desolvation and ionization of GIPCs can give the appearance for more possible species than are actually present. To further characterize complex GIPCs, a MRM method was developed to identify different iterations of sugars. This allowed for identification of sugar composition by both mass and retention time. Using this method, two unique complex GIPCs, (Pent) ${ }_{3}-\mathrm{Hex}-\mathrm{Hex}(\mathrm{NAc})-\mathrm{HexA}-$ IPC (Series E) and (Pent) $)_{2}$-Hex-Hex(NAc)-HexA-IPC (Series D) were identified. Neither of 
these species was found in leaf, though both forms of GIPC (lacking the $N$-acetyl or NAc substitution) was previously reported in Arabidopsis cell culture (Buré et al., 2011). The differences in observed retention times indicate that both of these species are found in planta and are not formed as a result of in source fragmentation. The presence of $\mathrm{N}$-acetylated hexose or Hex(NAc) containing-GIPCs has recently been reported in small amounts in Arabidopsis seedlings and seeds (Tellier et al., 2014), and GIPCs containing complex sugars have been reported in Arabidopsis cell culture (Buré et al., 2011). Our results for pollen differ from those in that $\mathrm{Hex}$ (NAc) containing-GIPCs were detected on the pentose-containing GIPCs, but not in Arabidopsis cell cultures (Buré et al., 2011), and no pentose-containing GIPCs were found in Arabidopsis seedlings and seeds (Tellier et al., 2014). Provisionally identified Hex-Hex-HexAIPCs (Series B) in leaf tissue, but not in pollen, were also found which contrasts with the previous detection of Hex-Hex $(\mathrm{OH})$-HexA-IPCs (Series B) in Arabidopsis cell culture but not in leaf (Buré et al., 2011). This finding builds upon recent work by Tellier et al., 2014, in profiling different organs to identify unique Arabidopsis sphingolipids. The implication of these findings is that different sphingolipid structures may be required for optimal function in different tissues, suggesting that either the tissue environment requires a modified sphingolipid structure to perform the same function carried out in other tissues, or that a modified function is demanded of the different sphingolipid structure. Future studies to modify GIPC structure may help shed light on the role of GIPC structure in different tissue types.

Publicly available RNA-Seq (Loraine et al., 2013) and microarray (Winter et al., 2007) data also pointed to distinct sphingolipid-related gene expression profiles. Consistent with the high GlcCer content, the GCS gene encoding the GlcCer synthase, which catalyzes the final step in GlcCer synthesis, was at least 100-fold more highly expressed in pollen than in seedlings and leaves. In addition, the $\mathrm{LOH} 2$ gene for the Type I ceramide synthase, which generates $\mathrm{C} 16$ fatty acid-containing ceramides for GlcCer synthesis, was more highly expressed in pollen based on microarray data and confirmed by qPCR analyses. Furthermore, in addition to the expected nearly exclusive expression of the $\triangle 4 D E S$ gene in pollen relative to seedlings and leaves, genes for other LCB modification enzymes was more highly expressed in pollen. Among these genes are $S L D 1$ and $S L D 2$, encoding the LCB $\triangle 8$ desaturase, and $S B H 1$ and $S B H 2$, encoding the LCB $\mathrm{C}-4$ hydroxylase. Interestingly, the $S L D 2$ and $S B H 2$ genes were more highly expressed in pollen than $S L D 1$ and $S B H 1$, respectively. By contrast, $S L D 1$ and $S B H 1$ are more highly expressed than 
SLD2 and SBH2 in leaves and seedlings, based on data from RNA-Seq and microarrays and from published northern blot analyses (Chen et al., 2012; Chen et al., 2008). These findings suggest that the $S L D 2$ and $S B H 2$ may have distinct and important roles in sphingolipid biosynthesis in pollen, including supporting biosynthetic pathways for GlcCer.

The primary goal of this study was to detect differences in sphingolipid metabolism between Arabidopsis pollen and leaf using lipidomic profiling and gene expression data. Although the analytical methods used are incapable of determining the exact identities of GIPC sugar residues and their linkages, they do provide an intriguing documentation of the specialized localization of novel GIPC species in Arabidopsis pollen that warrants further phytochemical investigation. Indeed, two of the major questions left in plant sphingolipid research are the function and identity of the variety of GIPC structures in plant physiology. The use of ESIMS/MS and the application of multiple reaction monitoring (MRM) offer unprecedented sensitivity for identifying and reproducibly profiling the general classes of sugar residues in GIPCs with as little as three mg of tissue (Markham and Jaworski, 2007). In addition, with internal standards, ESI-MS/MS coupled with MRM enable quantification of GIPCs as now routinely done for the major Arabidopsis leaf GIPC (Markham and Jaworski, 2007). Notably, the only GIPC head group structures that have been completely characterized are those from tobacco leaf based on research from Lester and coworkers (Hsieh et al., 1978; Hsieh et al., 1981; Kaul and Lester, 1975; Kaul and Lester, 1978). These characterizations were conducted using extracts from three $\mathrm{kg}$ of tobacco leaf and established that glucosamine (+/- $\mathrm{N}$-acetylation; $\alpha$ $1 \rightarrow 4)$ glucuronic acid $(\alpha 1 \rightarrow 2)$ myo-inositol-1-O-phosphorylceramide are the major GIPC forms in tobacco leaves. From these studies, it can be inferred that the major GIPC of Arabidopsis pollen is possibly $N$-acetylated-glucosamine $(\alpha 1 \rightarrow 4)$ glucuronic acid $(\alpha 1 \rightarrow 2)$ myo-inositol-1-Ophosphoceramide (Fig. 7), and the other Arabidopsis pollen GIPCs likely arise from additional and alternative glycosylation of the glucuronic acid $(\alpha 1 \rightarrow 2)$ myo-inositol-1-O-phosphoceramide core structure. However, detailed structural characterization of Arabidopsis GIPCs awaits further purification and structural characterization. Given that these analyses require considerable amounts of plant material, complete structural elucidation of the distinct GIPCs of Arabidopsis pollen will be especially challenging using current approaches.

\section{Concluding remarks}


The findings herein suggest that sphingolipid metabolism is strikingly different in Arabidopsis pollen than in leaves. In fact, based on compositional similarities, sphingolipid metabolism in Arabidopsis pollen is more similar to that found in leaves of plants such as tomato, soybean and tobacco (Buré et al., 2011; Hsieh et al., 1981; Kaul and Lester, 1978; Markham et al., 2006). Similar to Arabidopsis pollen, previous studies, for example, have shown that GlcCer of tomato and soybean leaves is not only enriched in d18:2 LCBs, but also GlcCer concentrations are equal or greater than GIPC concentrations, suggesting a correlation between d18:2 and GlcCer concentrations in plant tissues (Markham et al., 2006). In addition, the large complexity of GIPC head groups in Arabidopsis pollen, in contrast to leaves, is similar to that reported for tobacco leaves (Hsieh et al., 1981; Kaul and Lester, 1978). Given the GIPC structural dichotomy between Arabidopsis pollen and leaves, it is possible that a comparison of glycosyltransferase gene expression levels between these organs may reveal novel pollenspecific genes associated with complex GIPC head group assembly. Overall, our findings point to specialization in sphingolipid metabolism in pollen leading to distinct sphingolipid composition, the functional significance of which remains to be elucidated.

\section{Experimental}

\subsection{Pollen isolation}

Pollen was isolated from Arabidopsis Col-0 and $\Delta 4$ desaturase mutant (Salk_107761.42.15.x) plants grown in $16 \mathrm{~h}$ days at $22^{\circ} \mathrm{C}$. Flowers were harvested from five week old plants and incubated with shaking for $2 \mathrm{~min}$ in $0.3 \mathrm{M}$ mannitol as previously described (Honys and Twell, 2003). Pollen was collected by centrifugation at $3780 \mathrm{x}$ g for $10 \mathrm{~min}$ in $50 \mathrm{~mL}$ aliquots. Pollen pellets were then pooled in a microcentrifuge tube and collected by centrifugation at $16.3 \times 1000$ $\mathrm{g}$ for $5 \mathrm{~min}$. Isolated pollen was flash frozen in liq $\mathrm{N}_{2}$ and stored at $-80^{\circ} \mathrm{C}$.

\subsection{Sphingolipidomic analysis}

Sphingolipids were extracted from 1 to $2 \mathrm{mg}$ of lyophilized pollen using the lower phase of iPrOH/hexane/water (55:20:25 v/v/v) followed by $\mathrm{Me}_{3} \mathrm{~N}: \mathrm{H}_{2} \mathrm{O}(33: 67, \mathrm{v} / \mathrm{v})$ treatment described previously (Markham and Jaworski, 2007). Samples were dissolved in tetrahydrofuran $(\mathrm{THF}) / \mathrm{MeOH} / \mathrm{H}_{2} \mathrm{O}(2: 1: 2 \mathrm{v} / \mathrm{v} / \mathrm{v})$ containing $0.1 \% \mathrm{HCO}_{2} \mathrm{H}$. Sphingolipids were analyzed using a 
Shimadzu Prominence UPLC coupled with a QTRAP4000 mass spectrometer (ABSciex) as previously described (Kimberlin et al., 2013). MRMs to initially detect N-acetyl-sugarcontaining GIPCs were calculated by adding 41 mass units to the Q1 ion of previously described GIPC MRMs (Markham and Jaworski 2007). Instrument potentials and chromatography conditions for the initial detection of N-acetyl-sugar-containing GIPCs were as for Hex-HexAGIPCs described previously (Kimberlin et al., 2013). Precursor ion scanning to assess GIPC modifications was performed by monitoring for the t18:1_h24:1 backbone (precursors of $m / z$ 662.6) combined with chromatographic separation of GIPCs as described (Kimberlin et al., 2013).

\subsection{Complex GIPC multiple reaction monitoring method}

Pollen sphingolipid extracts were injected onto a reversed phase $75 \mathrm{~mm}$ Kinetex C18 HPLC column and eluted with a binary gradient with a flow rate of $0.60 \mathrm{~mL} / \mathrm{min}$ with a column temperature of $40^{\circ} \mathrm{C}$. The specific source and gradient conditions used can be found in Supplemental Tables 2 and 3, respectively. The mass spectrometer was set to record starting at minute 2 and continued to minute 14. The mass spectrometer was operated in positive electrospray ionization using multiple reaction monitoring (MRM). GIPC structures monitored and their corresponding MRMs can be found in Supplemental Table 4 along with the declustering potentials and collision energies used.

\subsection{Expanded LC-ESI/MS/MS profiling method modifications}

Sphingolipid profiling to monitor an expanded set of sphingolipid structures was done using modifications of chromatography conditions and instrument settings, building on those described previously by Markham and Jaworski (2007). The QTRAP4000 ion spray voltage, entrance potential, and collision exit potential were set to $5000 \mathrm{~V}, 10 \mathrm{~V}$, and $14 \mathrm{~V}$ respectively. For free

LCB analysis, the collision exit potential was set at $17 \mathrm{~V}$. Curtain gas, gas 1 , and gas 2 were set to $20 \mathrm{psi}, 60 \mathrm{psi}$, and 50 psi respectively for all classes except for hydroxyceramide analysis, which used curtain gas at 10 psi, gas 1 at 40 psi and gas 2 at 50 psi. A reversed phase $100 \mathrm{~mm}$ Acclaim C-18 HPLC column (ThermoScientific, Waltham, MA USA) was eluted by a binary gradient formed by buffers $\mathrm{A}$ and $\mathrm{B}$ described above with a flow rate of $1.00 \mathrm{~mL} / \mathrm{min}$ with a column temperature of $40^{\circ} \mathrm{C}$ for ceramide, hydroxyceramide and GlcCer elution gradients. For 
provisionally identified $\operatorname{Hex}(\mathrm{OH})-\mathrm{HexA}$ GIPCs and free LCB gradients, the flow rate was set at $0.8 \mathrm{ml} / \mathrm{min}$ Source temperatures used were $550 \mathrm{C}$ (ceramides), $300^{\circ} \mathrm{C}$ (hydroxyceramides), $350^{\circ}$ $\mathrm{C}$ (GlcCer), $350^{\circ} \mathrm{C}(\mathrm{Hex}(\mathrm{OH})-\mathrm{HexA}-\mathrm{GIPC})$, and $400^{\circ} \mathrm{C}$ (free LCB). A summary of these conditions can be found in Supplemental Table 2. Binary gradient percentages and time monitored can be found in Supplemental Table 3. MRMs along with the corresponding collision energies and declustering potentials can be found in Supplemental Table 4.

\subsection{RNA-Seq and microarray data mining}

Previously published RNA-Seq data (Loraine et al., 2013) comparing pollen and seedling was mined for sphingolipid synthesis genes. Data were compiled from RPM normalized data for genes encoding the following polypeptides: SPT subunits LCB1 (At4g36480), LCB2a (At5g23670), LCB2b (At3g48780), the two small subunits of SPT (ssSPT) ssSPTa_(At1g06515) and ssSPTb (At2g30942), the two 3-ketosphinganine reductases TSC10A (At5g19200) and TSC10B (At3g06060), the two sphingoid base C-4 hydroxylases SBH1 (At1g69640) and SBH2 (At1g14290), the ceramide synthases LOH1 (At3g25540), LOH2 (At3g19260), and LOH3 (At1g13580), LCB $\Delta 4$ desaturase (At4g04930), the two LCB $\Delta 8$ desaturases SLD1 (At3g61580) and SLD2 (At2g46210), glucosylceramide synthase (GCS;At2g19880), the three inositolphosphoceramide synthases (IPS) IPS1 (At3g54020), IPS2 (At2g37940), and IPS 3 (At2g29525) and the UDP-glucose IPC transferase IPUT1 (At5g18480). Microarray data for Arabidopsis sphingolipid genes were obtained from the Arabidopsis eFP Browser 2.0 (Winter et al., 2007). Gene numbers, names, and probe sets can be found in Supplemental Table 5. Vegetative rosette and mature pollen tissues were compared using total expression values. Error is represented by the reported standard deviation of three experiments.

\subsection{RT-PCR and qPCR}

For analyses of organ-specific expression of 4, 6- to 8-week old Col-0 plants were used as sources of plant material. Pollen was harvested as described previously (Johnson-Brousseau and McCormick, 2004). RNA extraction was performed using the RNeasy Plant Kit (Qiagen) according to the manufacturer's protocol. RNA $(1 \mu \mathrm{g})$ was treated with DNase I (Invitrogen) according to the manufacturer's protocol. Treated RNA was then reverse transcribed to cDNA with the iScript cDNA synthesis kit (Bio-Rad) according to the manufacturer's protocol. RT- 
PCR was conducted with an annealing temperature of $56^{\circ} \mathrm{C}$ for 40 cycles. Forward and reverse primers used for $\triangle 4 D E S$ were 5-GAGGACGTGAGAAGATATCATC-3 and 5-

GCAAGGTTGTGACTTAGCTCATG-3. Forward and reverse primers used for the control ubiquitin-conjugating enzyme At5g25760 were 5-ATGCAGGCATCAAGAGCGCGACTGT-3 and 5-CACCGCCTTCGTAAGGAGTCTCCGA-3. qPCR was performed on the cDNA using the Bio-Rad MyiQ iCycler qPCR instrument. SYBR green was used as the fluorophore in a qPCR supermix (Qiagen). QuantiTect (Qiagen) primer sets for LOH2 (QT00774949) were used for relative quantification with PP2AA3 (At1g13320) used as an internal reference gene.

\subsection{Pollen imaging}

Pollen imaging was performed using an Olympus AX70 optical microscope. Anthers and siliques of mature plants were isolated using a Nikon SMZ745T dissection microscope. Anthers were smeared on a glass slide and incubated with Alexander stain (Alexander, 1969) at $4{ }^{\circ} \mathrm{C}$ for 45 min before viewing. Pollen viability was assessed by shape and color.

\section{Acknowledgements}

The research was supported by funding from the U.S. National Science Foundation (MCB1158500) to EBC. 


\section{Figure Legends}

Figure 1: Viability staining of Col-0 pollen collected (A) directly from anther and (B) after isolation in 0.3 M mannitol. The presence of round, dark purple pollen in $\mathbf{A}$ indicates that pollen was viable prior to extraction in mannitol. As shown in $\mathbf{B}$, the mannitol-extract is highly enriched in viable pollen but also contains minor amounts of unstained lysed pollen and anther debris. RT-PCR of the pollen specific $\triangle 4$ DES and loading control gene (At5g25760) on (C) Col-0 and (D) $\triangle 4$ DES mutant plants.

Figure 2: Sphingolipid profiles of glucosylceramides (GlcCer), provisionally identified glycosylinositolphosphoceramides ( $\mathrm{Hex}(\mathrm{OH})$-HexA-IPCs, Series A), ceramides, hydroxyceramides, and free LCBs for enriched pollen from Col-0 and LCB $\Delta 4$ desaturase knockout mutant ( $\triangle 4$ DES KO) obtained by ESI-LC MS/MS analyzes. All values shown as the average of measurements of three independent pollen isolations \pm SD.

Figure 3: ESI-MS/MS spectrum of ions detected by precursor $m / z 662.6$ scanning of GIPC species eluted between 5 and 10 minutes during chromatography as described in Kimberlin et al (2013). These spectra depict GIPC species built upon a t18:1_h24:1 ceramide backbone and show differences in pollen and leaf GIPC glycosylation patterns. (A) Abundant ions detected in leaf were $m / z$ 1099.2 (HexA-IPC), $m / z 1261.2$ (Hex(OH)-HexA-IPC, Series A). Further glycosylation by addition of a pentose sugar adds 132 mass units, while addition of a hexose sugar adds 162 mass units. Relatively small amounts of $m / z 1393.0$ (Pent-Hex(OH)-HexA-IPC), m/z 1423.1 (Hex-Hex(OH)-HexA-IPC, Series B), m/z 1555.2 (Pent-Hex-Hex(OH)-HexA-IPC, Series C) and $m / z 1687.2$ (Pent $2-H e x-H e x(O H)-H e x A-I P C$, Series D) were also detected in leaf. (B) GIPC species detected in pollen contain $\operatorname{Hex}(\mathrm{OH})$ species found in leaf as well as Hex(NAc) species: $m / z$ 1302.2 (Hex(NAc)-HexA-IPC, Series A), m/z 1464.6 (Hex-Hex(NAc)-HexA-IPC, Series B), $m / z$ 1596.4 (Pent-Hex-Hex(NAc)-HexA-IPC, Series C), m/z 1728.0 (Pent 2 -HexHex(NAc)-HexA-IPC, Series D) and $m / z 1860.4$ (Pent ${ }_{3}$-Hex-Hex(NAc)-HexA-IPC, Series E). Hex, hexose; Pent, pentose; HexA, hexuronic acid; $\operatorname{Hex}(\mathrm{OH})$, hexose lacking $N$-acetylation; Hex(NAc), hexose with $N$-acetylation; IPC, inositolphosphoceramide. 
Figure 4: Detection of provisionally identified complex GIPC species in Arabidopsis Col-0. LC-MSMS traces are shown for different complex GIPC species (A) Hex(NAc)-HexA-IPC

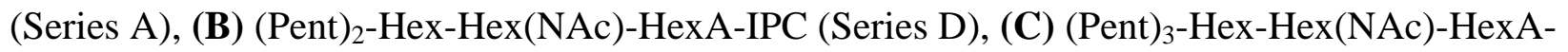
IPC (Series E), (D) Hex-Hex $(\mathrm{OH})-H e x A-I P C ~(S e r i e s ~ B)$ and their relative amounts in pollen and leaf. All head groups shown are bound to a t18:1_h24:1 ceramide backbone except for the Hex$\operatorname{Hex}(\mathrm{OH})-H e x A-I P C$ (Series B) which is built upon the t18:1_h24:0 ceramide backbone.

Figure 5: (A) Total sphingolipid per class in pollen identified by LC-MS/MS. Data represented as the average of three independent pollen isolations \pm SD. (B) Total amounts of each LCB found in both Col-0 and $\triangle 4 D E S$ mutant pollen. Data represents the average of the sum of all LCB levels from three independent pollen isolations with standard deviation (C) Total sphingolipid identified by LC-MS/MS. Data represents the average of the sum of all sphingolipid species \pm SD.

Figure 6: Arabidopsis RNA-Seq gene expression levels for different genes involved with sphingolipid synthesis in pollen and seedling. Data were compiled from RPM normalized data (Loraine et al., 2013) for genes encoding the following polypeptides: SPT subunits LCB1 (At4g36480), LCB2a (At5g23670), LCB2b (At3g48780), the two small subunits of SPT (ssSPT) ssSPTa_(At1g06515) and ssSPTb (At2g30942), the two 3-ketosphinganine reductases TSC10A (At5g19200) and TSC10B (At3g06060), the two sphingoid base C-4 hydroxylases SBH1 (At1g69640) and SBH2 (At1g14290), the ceramide synthases LOH1 (At3g25540), LOH2 (At3g19260), and LOH3 (At1g13580), LCB $\Delta 4$ desaturase (At4g04930), the two LCB $\Delta 8$ desaturases SLD1 (At3g61580) and SLD2 (At2g46210), glucosylceramide synthase (GCS; At2g19880), the three IPC synthases (IPS) IPS1 (At3g54020), IPS2 (At2g37940), and IPS3 (At2g29525), the UDP-glucose IPC transferase IPUT1 (At5g18480).

Figure 7: Inferred structure of the major GIPC species in Arabidopsis pollen. The structure shown is $N$-acetylated-glucosamine $(\alpha 1 \rightarrow 4)$ glucuronic acid $(\alpha 1 \rightarrow 2)$ myo-inositol-1-Ophosphoceramide, based on tobacco GIPC structural characterizations conducted by Lester and coworkers (Hsieh et al., 1978; Hsieh et al., 1981; Kaul and Lester, 1975; Kaul and Lester, 1978). 
The other Arabidopsis pollen GIPCs likely arise from additional and alternative glycosylation of the glucuronic acid $(\alpha 1 \rightarrow 2)$ myo-inositol-1-O-phosphorylceramide core structure.

\section{References}

Borner, G. H., Sherrier, D., Weimar, T., Michaelson, L., Hawkins, N., Macaskill, A., Napier, J., Beale, M., Lilley, K., Dupree, P., 2005. Analysis of detergent-resistant membranes in Arabidopsis. Evidence for plasma membrane lipid rafts. Plant Physiol. 137, 104-116.

Brodersen, P., Petersen, M., Pike, H., Olszak, B., Skov, S., Odum, N., Jørgensen, L., Brown, R., Mundy, J., 2002. Knockout of Arabidopsis accelerated-cell-death11 encoding a sphingosine transfer protein causes activation of programmed cell death and defense. Genes \& Development 16, 490-502.

Buré, C., Cacas, J.-L., Wang, F., Gaudin, K., Domergue, F., Mongrand, S., Schmitter, J.-M., 2011. Fast screening of highly glycosylated plant sphingolipids by tandem mass spectrometry. Rapid Comm. Mass Spec. 25, 3131-3145.

Buré, C., Cacas, J. L., Mongrand, S., Schmitter, J. M., 2014. Characterization of glycosyl inositol phosphoryl ceramides from plants and fungi by mass spectrometry. Anal. Bioanal. Chem. 406, 995-1010.

Cacas, J. L., Buré, C., Furt, F., Maalouf, J. P., Badoc, A., Cluzet, S., Schmitter, J. M., Antajan, E., Mongrand, S., 2013. Biochemical survey of the polar head of plant glycosylinositolphosphoceramides unravels broad diversity. Phytochemistry 96, 191-200.

Cantrel, C., Vazquez, T., Puyaubert, J., Reze, N., Lesch, M., Kaiser, W. M., Dutilleul, C., Guillas, I., Zachowski, A., Baudouin, E., 2011. Nitric oxide participates in cold- 
responsive phosphosphingolipid formation and gene expression in Arabidopsis thaliana. New Phytologist 189, 415-427.

Chen, M., Cahoon, E.B., Saucedo-García, M., Plasencia, J., Gavilanes-Ruíz, M., 2010. Plant Sphingolipids: Structure, Synthesis and Function. In: Wada, H., Murata, N. (Eds.), Lipids in Photosynthesis, vol. 30. Springer Netherlands, pp. 77-115.

Chen, M., Han, G., Dietrich, C. R., Dunn, T. M., Cahoon, E. B., 2006. The essential nature of sphingolipids in plants as revealed by the functional identification and characterization of the Arabidopsis LCB1 subunit of serine palmitoyltransferase. Plant Cell 18, 3576-3593.

Chen, M., Markham, J. E., Cahoon, E. B., 2012. Sphingolipid $\Delta 8$ unsaturation is important for glucosylceramide biosynthesis and low-temperature performance in Arabidopsis. Plant J. 69, 769-781.

Chen, M., Markham, J. E., Dietrich, C. R., Jaworski, J. G., Cahoon, E. B., 2008. Sphingolipid long-chain base hydroxylation is important for growth and regulation of sphingolipid content and composition in Arabidopsis. Plant Cell 20, 1862-1878.

Coursol, S., Fan, L. M., Le Stunff, H., Spiegel, S., Gilroy, S., Assmann, S. M., 2003. Sphingolipid signalling in Arabidopsis guard cells involves heterotrimeric G proteins. Nature 423, 651-654.

Coursol, S., Le Stunff, H., Lynch, D., Gilroy, S., Assmann, S., Spiegel, S., 2005. Arabidopsis sphingosine kinase and the effects of phytosphingosine-1-phosphate on stomatal aperture. Plant Physiol. 137, 724-737.

Dietrich, C., Han, G., Chen, M., Berg, R., Dunn, T., Cahoon, E., 2008. Loss-of-function mutations and inducible RNAi suppression of Arabidopsis LCB2 genes reveal the critical role of sphingolipids in gametophytic and sporophytic cell viability. Plant J. 54, 284-298.

Guillas, I., Guellim, A., Reze, N., Baudouin, E., 2012. Long chain base changes triggered by a short exposure of Arabidopsis to low temperature are altered by AHb1 non-symbiotic haemoglobin overexpression. Plant Physiol. Biochem. 63C, 191-195.

Honys, D., Twell, D., 2003. Comparative analysis of the Arabidopsis pollen transcriptome. Plant Physiol.132, 640-652. 
Hsieh, T. C., Kaul, K., Laine, R. A., Lester, R. L., 1978. Structure of a major glycophosphoceramide from tobacco leaves, PSL-I: 2-deoxy-2-acetamido-Dglucopyranosyl $(\alpha 1 \rightarrow 4)$-D-glucuronopyranosyl $(\alpha 1 \rightarrow 2)$ myoinositol-1-Ophosphoceramide.Biochemistry 17, 3575-3581.

Hsieh, T. C., Lester, R. L., Laine, R. A., 1981. Glycophosphoceramides from plants. Purification and characterization of a novel tetrasaccharide derived from tobacco leaf glycolipids. J. Biol. Chem. 256, 7747-7755.

Imai, H., Yamamoto, K., Shibahara, A., Miyatani, S., Nakayama, T., 2000. Determining doublebond positions in monoenoic 2-hydroxy fatty acids of glucosylceramides by gas chromatography-mass spectrometry. Lipids 35, 233-236.

Islam, M. N., Jacquemot, M. P., Coursol, S., Ng, C. K., 2012. Sphingosine in plants--more riddles from the Sphinx? New Phytologist 193, 51-57.

Johnson-Brousseau, S. A., McCormick, S., 2004. A compendium of methods useful for characterizing Arabidopsis pollen mutants and gametophytically- expressed genes. Plant J. 39, 761-775.

Kaul, K., Lester, R. L., 1975. Characterization of inositol-containing phosphosphingolipids from tobacco leaves: Isolation and identification of two novel, major lipids: $N$ acetylglucosamidoglucuronidoinositol phosphorylceramide and glucosamidoglucuronidoinositol phosphorylceramide. Plant Physiol 55, 120-129.

Kaul, K., Lester, R. L., 1978. Isolation of six novel phosphoinositol-containing sphingolipids from tobacco leaves. Biochem. 17, 3569-3575.

Kimberlin, A., Majumder, S., Han, G., Chen, M., Cahoon, R., Stone, J., Dunn, T., Cahoon, E., 2013. Arabidopsis 56-amino acid serine palmitoyltransferase-interacting proteins stimulate sphingolipid synthesis, are essential, and affect mycotoxin sensitivity. Plant Cell 25, 4627-39

Loraine, A. E., McCormick, S., Estrada, A., Patel, K., Qin, P., 2013. RNA-Seq of Arabidopsis pollen uncovers novel transcription and alternative splicing. Plant Physiol. 162, 1092 1109. 
Lynch, D. V., Dunn, T. M., 2004. An introduction to plant sphingolipids and a review of recent advances in understanding their metabolism and function. New Phytologist 161, 677-702.

Markham, J., Jaworski, J., 2007. Rapid measurement of sphingolipids from Arabidopsis thaliana by reversed-phase high-performance liquid chromatography coupled to electrospray ionization tandem mass spectrometry. Rapid Comm. Mass Spec. 21, 1304-1314.

Markham, J. E., Li, J., Cahoon, E. B., Jaworski, J. G., 2006. Separation and identification of major plant sphingolipid classes from leaves. J.Biol. Chem. 281, 22684-22694.

Markham, J. E., Molino, D., Gissot, L., Bellec, Y., Hematy, K., Marion, J., Belcram, K., Palauqui, J. C., Satiat-Jeunemaitre, B., Faure, J. D., 2011. Sphingolipids containing verylong-chain fatty acids define a secretory pathway for specific polar plasma membrane protein targeting in Arabidopsis. Plant Cell 23, 2362-2378.

Michaelson, L. V., Zauner, S., Markham, J. E., Haslam, R. P., Desikan, R., Mugford, S., Albrecht, S., Warnecke, D., Sperling, P., Heinz, E., Napier, J. A., 2009. Functional characterization of a higher plant sphingolipid $\Delta 4$-desaturase: defining the role of sphingosine and sphingosine-1-phosphate in Arabidopsis. Plant Physiol. 149, 487-498.

Mongrand, S., Morel, J., Laroche, J., Claverol, S., Carde, J.-P., Hartmann, M.-A., Bonneu, M., Simon-Plas, F., Lessire, R., Bessoule, J.-J., 2004. Lipid rafts in higher plant cells: purification and characterization of Triton X-100-insoluble microdomains from tobacco plasma membrane. J. Biol. Chem. 279, 36277-36286.

Mortimer, J., Yu, X., Albrecht, S., Sicilia, F., Huichalaf, M., Ampuero, D., Michaelson, L., Murphy, A., Matsunaga, T., Kurz, S., Stephens, E., Baldwin, T., Ishii, T., Napier, J., Weber, A., Handford, M., Dupree, P., 2013. Abnormal glycosphingolipid mannosylation triggers salicylic acid-mediated responses in Arabidopsis. Plant Cell 25, 1881-1894.

Nagano, M., Ishikawa, T., Ogawa, Y., Iwabuchi, M., Nakasone, A., Shimamoto, K., Uchimiya, H., Kawai-Yamada, M., 2014. Arabidopsis Bax inhibitor-1 promotes sphingolipid synthesis during cold stress by interacting with ceramide-modifying enzymes. Planta 240, 77-89.

Ng, C. K., Carr, K., McAinsh, M. R., Powell, B., Hetherington, A. M., 2001. Drought-induced guard cell signal transduction involves sphingosine-1-phosphate. Nature 410, 596-599. 
Rennie, E. A., Ebert, B., Miles, G. P., Cahoon, R. E., Christiansen, K. M., Stonebloom, S., Khatab, H., Twell, D., Petzold, C. J., Adams, P. D., Dupree, P., Heazlewood, J. L., Cahoon, E. B., Scheller, H. V., 2014. Identification of a sphingolipid $\alpha-$ glucuronosyltransferase that is essential for pollen function in Arabidopsis. Plant Cell 26, 3314-3325.

Saucedo-García, M., Guevara-García, A., González-Solís, A., Cruz-García, F., Vázquez-Santana, S., Markham, J., Lozano-Rosas, M., Dietrich, C., Ramos-Vega, M., Cahoon, E., Gavilanes-Ruíz, M., 2011. MPK6, sphinganine and the LCB2a gene from serine palmitoyltransferase are required in the signaling pathway that mediates cell death induced by long chain bases in Arabidopsis. New Phytologist 191, 943-957.

Sperling, P., Franke, S., Luthje, S., Heinz, E., 2005. Are glucocerebrosides the predominant sphingolipids in plant plasma membranes? Plant Physiol. Biochem. 43, 1031-1038.

Sperling, P., Zahringer, U., Heinz, E., 1998. A sphingolipid desaturase from higher plants. Identification of a new cytochrome b5 fusion protein. J. Biol. Chem. 273, 28590-28596.

Tellier, F., Maia-Grondard, A., Schmitz-Afonso, I., Faure, J. D., 2014. Comparative plant sphingolipidomic reveals specific lipids in seeds and oil. Phytochemistry 103, 50-58.

Teng, C., Dong, H., Shi, L., Deng, Y., Mu, J., Zhang, J., Yang, X., Zuo, J., 2008. Serine palmitoyltransferase, a key enzyme for de novo synthesis of sphingolipids, is essential for male gametophyte development in Arabidopsis. Plant Physiol. 146, 1322-1332.

Winter, D., Vinegar, B., Nahal, H., Ammar, R., Wilson, G. V., Provart, N. J., 2007. An "Electronic Fluorescent Pictograph" browser for exploring and analyzing large-scale biological data sets. PloS One 2, e718. 